\title{
Conceptualizing and Measuring Group Openness and Cohesion as Dimensions of Group Personality
}

\author{
Marius Deckers, Tobias Altmann, Marcus Roth \\ Department of Differential Psychology, University of Duisburg-Essen, Essen, Germany \\ Email: marius.deckers@uni-due.de
}

How to cite this paper: Deckers, M., Altmann, T., \& Roth, M. (2018). Conceptualizing and Measuring Group Openness and Cohesion as Dimensions of Group Personality. Psychology, 9, 80-100.

https://doi.org/10.4236/psych.2018.91006

Received: December 8, 2017

Accepted: January 9, 2018

Published: January 12, 2018

Copyright (๑) 2018 by authors and Scientific Research Publishing Inc. This work is licensed under the Creative Commons Attribution International License (CC BY 4.0).

http://creativecommons.org/licenses/by/4.0/

\section{(c) (i) Open Access}

\begin{abstract}
Groups exhibit behavioral consistencies similar to individuals, such as making more or less bold decisions or struggling more or less frequently. However, previous research findings focus only on specific dimensions of groups instead of the general structure of group personality. Based on previous research findings on group and team efficiency, we derived two basic dimensions of group personality, group openness and cohesion, representing the group's outward and inward orientation, respectively. We present the "Group Openness and Cohesion Questionnaire" (GOCQ) as a measure to assess these two group personality dimensions in groups independent of their context. Confirmatory factor analysis confirmed the proposed two-dimensional structure with good to excellent psychometric properties. Evidence of validity is provided through group age, group size, and by the fact that the ratings reflect team characteristics. In general, the results support the conceptualization of these two basic dimensions of group personality and provide first indications of validity of the presented measure GOCQ.
\end{abstract}

\section{Keywords}

Personality, Group, Cohesion, Openness, Questionnaire

\section{Introduction}

The concept of personality is usually only applied to individuals and defined by different traits that are intra-individually consistent across situations and stable over time, such as the Big Five of the Five-Factor model (McCrae \& Costa Jr., 1999). However, whenever several individuals join together to form a group, we find that this group as a new entity also begins to exhibit aspects of coherent 
believes, perceptions, and behavioral styles. Thus, the group acts in ways that are consistent across situations and stable over time (Dang \& Ilgen, 2006), and therefore possesses what might be called group personality.

Historically, personality dimensions are conceptualized as behavioral tendencies of individuals on a latent level that are consistent across situations and stable over time. As they are related to behavior and cognition, individual personality dimensions are largely based on the way individuals interact with and perceive the world and themselves. This is reflected in the content of the items that personality dimensions are assessed with, for example "I would be quite bored by a visit to an art gallery" or "I often push myself very hard when trying to achieve a goal' (Ashton \& Lee, 2007).

With our goal to conceptualize group personality dimensions, another level is added to the concept of interaction with and perceiving the world. When viewing the group as the entity that a personality dimension is assigned to, one needs to consider the fact that the group members not only interact with the world outside of the group, but also with the other group members. This means that group personality dimensions should take both the interactions of the group members as well as the interactions the entire group has with its environment into consideration. Groups need to define themselves in regard to how they behave and think inwardly and outwardly and group personality dimensions should reflect that.

The term group personality dimension, as it is used here, refers to a comprehensive group trait that describes very generalized behavioral tendencies of groups. It is not meant to replace group traits as they are researched in social psychology and organizational psychology. It originates from the perspective of personality psychology that aims to find the smallest number of superordinate constructs which account for the largest amount of behavioral tendencies in individuals in general. Because we apply this concept to the group level, the label "group personality" is used. Other authors describe the same aspect as "group climate" or "group culture". With the term "group personality" we want to emphasize the perspective from personality psychology, i.e., the behavioral aspect of the group interactions as well as the universal and comprehensive character of these dimensions.

In this paper, we propose that groups can be characterized by an overarching group personality structure that describes stable cognitive and behavioral patterns of a group as a whole and that is distinct from personality structures on the individual level. In the following, we briefly summarize the existing literature on both approaches to conceptualize group personality, suggest a bi-dimensional structure that is independent of specific group contexts, and present an inventory to assess these dimensions.

\subsection{Current Approaches to Group Personality}

There are basically two approaches to group personality in the literature so far: 
an indirect and a direct approach. The indirect approach argues that a group's personality is simply the average of the individual members' personalities. This approach, however, assumes without substantiation that the basic structure of group personality is the same as the basic structure of individual personality. In contrast, the direct approach assumes that the group is characterized by dimensions beyond those of individual personality. The main difference between the two approaches is that the indirect approach bases group personality on the personality of the individual members (which in turn is based on the behavior of the individual group members), while the direct approach bases group personality directly on the behavioral tendencies of the group as a whole. The latter approach is also referred to as group climate or sometimes as group culture (see below). Several approaches have been made to capture dimensions of group personality, however, to our knowledge, a coherent concept or theory of its fundamental structure is still missing.

\subsubsection{Indirect Approach}

The indirect approach to measuring group personality proposes that the personality traits of the individual group members alone can be used to calculate the group personality, with no further information about the group needed (Barrick, Stewart, Neubert, \& Mount, 1998). In the literature, there are three ways of doing such a calculation: mean score, mean score as well as variability, and extremes.

The first and most common way is to calculate a mean score of the values of each group member on a given personality trait. This method has been applied to personality traits such as extraversion, dominance (Heslin, 1964), and even intelligence (Williams \& Sternberg, 1988). This approach views group personality as a collective pool of a given characteristic that each group member contributes to. What is problematic about this type of approach is that personality characteristics are abstract constructs, and there is neither theory nor rationale as to why a group personality should be able to be calculated by means of individual personality score, and which behavioral tendencies on the group level it should result in.

The second way takes the variability of personality characteristics in the group into consideration as well (Barry \& Steward, 1997; Schneider, 1987). As differences between groups can be obstructed by calculating the mean only, comparing variances, ranges, and other proportions can help reveal those differences. For example, two groups might have the same mean conscientiousness score, but the trait is normally distributed in one group, while in the other it is bimodal, i.e., composed of two subgroups, one of which scores very high and the other very low on the trait. Therefore, comparing variance in individual traits (Jackson et al., 1991; Tsui, Egan, \& Oreilly, 1992) and assessing the proportion of group members exhibiting high parameter values on a certain trait (such as extraordinarily high extraversion; Barry \& Stewart, 1997) reveal influences that might be obstructed by using the mean score only. However, the rationale for these evalu- 
ations has not been defined sufficiently and methodological concerns remain, such as most importantly, the lack of an underlying theory explaining the link between individual traits and overarching group traits.

The third way, calculating extremes, looks at the highest or lowest individual trait value within a group, with the idea that individuals far from the average on a certain trait can significantly influence the behavior of an entire group (Kenrick \& Funder, 1988). For example, in assembly line work, the slowest worker influences the speed of the entire line, while in problem solving tasks, the input of the member with the highest general mental ability is essential to quickly solving the problem (Steiner, 1972). As these examples show, this way is based on highly specific conditions that do not apply to all groups equally.

In general, the indirect approach with its three subtypes shows a lack of theoretical underpinning and should be considered methodologically questionable. Most of the studies that used the indirect approach employ it using the FiveFactor Model of personality (McCrae \& Costa Jr., 1999) or selected sub-facets of it assessed at the individual level (Bear, Oldham, Jacobsohn, \& Hollingshead, 2008; Homan et al., 2008).

Findings in this research tradition yielded inconsistent results. As an example, studies trying to predict group performance from group personality composition have found that the personality dimensions of the Five-Factor Model (neuroticism, extraversion, agreeableness conscientiousness, and openness to experience) greatly differ in their predictive power depending how they were calculated at the group level, which types of groups were assessed and how group performance was operationalized (for an overview, see: Barrick, Stewart, Neubert, \& Mount, 1998; van Vianen \& De Dreu, 2001). However, in many studies, openness to experience emerged as a dimension with high predictive power for different group-level outcomes, such as group performance (Barrick \& Mount, 1991; Homan et al., 2008), group creativity (Bear et al., 2008) or within-group information exchange (Bond \& Shiu, 1997). Overall, openness to experience at the individual level seems to be the only personality dimension that can relatively consistently predict a wider range of group-level outcomes.

These findings in past research point towards a general importance of openness at the group level, regardless of the methodological and the theoretical difficulties associated with the indirect approach.

This lead us to conceptualizing group openness as the first key dimension of group personality that seems applicable to all groups independent of their context. Group openness describes a group's outward orientation, which entails in how far a group is willing to and interested in welcoming new ideas, impulses, principles, activities, and members. Just like personality dimensions at the individual level explain behavioral tendencies in individual persons, this dimension explains a behavioral tendency that can be observed in groups. However, a concept of openness at the group level also requires the measurement at the group level, instead of at the individual level. 


\subsubsection{Direct Approach}

The direct approach tries to conceptualize relevant dimensions of group personality by having the group members directly rate their respective group as a whole. Each member rates the whole group as one entity regarding, e.g., its effectiveness, creativity, etc. Systematic research in this field is primarily focused on the concept of climate, which has been assessed as work team climate (Anderson \& West, 1996), family climate (Bjornberg \& Nicholson, 2007; Kurdek \& Fine, 1993), or therapy group climate (Law et al., 2012).

Climate has been defined slightly differently in each context in which it has been assessed, which is most frequently in work organizations (as indicated by the large number of publications in this specific research area, as opposed to, for example, climate research in families or therapy groups). As Patterson et al. (2005) stated, the "dominant approach conceptualizes climate as employees' shared perceptions of organizational events, practices and procedures" (p. 380). In contrast to organizational culture, that primarily contains the perceived shared believes and values, organizational climate is more behaviorally oriented, describing patterns of interactions and behaviors in the organization (Schneider, 2000). With the focus on group personality, i.e., the stable and consistent cognitive and behavioral patterns of a group, group personality and climate appear naturally close to each other.

The most commonly used instrument for measuring work team climate is fittingly called the Team Climate Inventory (TCI) (Anderson \& West, 1998). In the TCI, team climate is divided into the following four factors: vision, participative safety, task orientation, and support for innovation. Vision refers to a collective motivating force for the work team through the presence of a clear, common, achievable goal. Participative safety describes the degree to which each group member can interact with the rest of the group without facing threats or judgement and while receiving trust and support. Task orientation refers to the extent to which the team is committed to good task performance and is willing to adjust and improve work practices to perform more efficiently. Support for innovation describes a general atmosphere of support for new and improved ideas for working processes within the team.

Such a measurement for facet-specific team climate (e.g., support for innovation) naturally falls short of being universally useful to describe all possible teams or groups in general. As mentioned by Schneider \& Reichers (1983), other facet-specific types of climate might be more useful in other team contexts where innovation is not the main concern, but when instead the team is striving for quality (e.g., health care providers) or change (e.g., a new political party). Even more so, people can form groups that are not work teams, e.g., societies and clubs, which require different measurements of climate. In sum, the direct approach so far only applies to specific teams and is hence mostly applied in organizational work team research (Anderson \& West, 1998; Cheng et al., 2013; Zhu et al., 2016).

In contrast to work teams, climate in families is conceptualized by the dimensions (emotional) warmth, conflict, supervision, and order (Family Climate In- 
ventory (FCI); Kurdek \& Fine, 1993); by acceptance, conflict, supervision, and autonomy granting in an updated version of the FCI (Kurdek, Fine, \& Sinclair, 1995); and by cohesion, process, and intergenerational interaction style (Family Climate Scales (FCS); Bjornberg \& Nicholson, 2007). All of these family climate concepts emphasize emotional connection, dealing with disagreement, and how the family is organized (which, in contrast to company teams, is not fixed in families). Because the FCI as the usual measure of choice for family climate was created to be answered by children, some authors interested in assessing the viewpoints of other family members derive or create their own measurements (Sbicigo \& Dell'Aglio, 2012). There does not seem to be a well-established international family climate questionnaire that is designed to be answered by the entire family. Furthermore, there is also no universally accepted family climate theory or concept-multiple authors have created their own questionnaires for their individual studies, each of which has different items and subscales depending on the specific research question and conditions.

Finally, therapy group climate (although rarely assessed, see Law et al., 2012) is conceptualized as consisting of engaged, conflict, and avoiding (Group Climate Questionnaire (GCQ); MacKenzie \& Tschuschke, 1993). Here again, climate in this context is composed of different subscales, but there are unfortunately very few publications on them.

These differences in climate definitions and subscales can likely be attributed to differences in values, goals, and desired outcomes in different kinds of groups. Overall, taking all these different climate constructs, definitions and subscales into consideration, the common denominator is the individual group members' feelings towards the group, the extent to which they feel close to each other, how much they trust each other, how much the group feels like a coherent entity to them, and the extent to which the group shares and agrees on a common goal. All of these facets can be subsumed by the term cohesion.

In the TCI (Anderson \& West, 1998), this facet is found in the participative safety subscale, which includes team members receiving trust and support from each other, and to a lesser extent in the vision subscale, which includes collective motivation and the presence of a common goal. In the first version of the FCI, the subscale (emotional) warmth includes the strength of the family members' emotional connection. This notion is also found in the second version of the FCI as part of the acceptance subscale, which includes perceived levels of emotional warmth and support (Kurdek et al., 1995). The FCS includes a cohesion subscale, which is subdivided into emotional cohesion, the strength of emotional connections among family members, and cognitive cohesion, the conformity of views on general topics within the family (Bjornberg \& Nicholson, 2007). In the GCQ, the subscale engaged describes a group climate of mutual trust and support, while the other subscales describe groups in which such a climate is absent (MacKenzie \& Tschuschke, 1993). Inventories that measure group constructs in other contexts (e.g. sports groups or school classes) often either include cohesion 
or are based on cohesion as well (Carron, Widmeyer, \& Brawley, 1985; Eys et al., 2009; Johnson \& Johnson, 1983).

Since cohesion appears to be a stable and consistent variable in climate inventories for groups in highly differing contexts, the concept can be assumed to constitute a general dimension of group personality. Although the conceptualization of cohesion differs to some extent depending on the context in which it is assessed and used (Carron \& Brawley, 2000), it always describes how individual members of a group feel like they belong to the group, how affectionate they are to one another, and to what extent the group shares a common goal. Cohesion is independent of which specific people form a group and of the group's goals, values, and outcomes. We therefore conceptualize it as the second dimension of group personality, forming the group's inward orientation, which consists of the group members' affection for the group, the degree to which they get along and how well they treat each other, as well as the degree to which they share and agree on a common goal.

In the past, instruments that included cohesion as a facet were always specifically created for specific teams or groups (such as work-related teams, sport teams, families, or therapy groups). There is not yet a concept nor measurement of cohesion that is comprehensive and therefore applicable to all groups independent of their context and setting. Corresponding to the concept of individual personality, in which all dimensions are applicable to all individuals in the general population, dimensions of group personality should also be applicable to all common types of groups.

\subsection{Hypotheses}

For the present research, we propose that group personality can be conceptualized with two dimensions, group openness and cohesion, as outlined above. Group openness refers to the extent to which a group is interested in and willing to incorporate new impulses, strategies, principles, methods, ideas and members, describing an outward orientation. Cohesion can be seen as the tendency of a group to feel like a cohesive entity, to exhibit mutual trust and affection among group members and to agree on and share a common goal, describing an inward orientation.

Since there currently is no inventory to measure group personality that is independent of specific contexts (e.g., organizational, sport, or family groups), we developed an inventory to measure this construct that includes group openness and cohesion.

For our first hypothesis, we expect that a structural model with the two factors group openness and cohesion will have an at least satisfactory model fit to the data obtained with the instrument. For our second hypothesis, we expect team membership to have a significant influence on factor-level answer patterns, so that being in a certain team will by itself will be predictive of answer patterns.

Concerning the validity measurements, we expect that the longer a group 
stays together, the higher its mean cohesion will be (third hypothesis), as cohesion can be expected to increase as group members know each other and interact frequently with each other over a longer period of time, as this will also likely increase how close they feel to each other (Harrison, Price, \& Bell, 1998). We further predict that the longer a group stays together, the higher its group openness will become (fourth hypothesis), because outside changes will be less likely to be perceived as a threat to the group and more likely to be perceived as an asset to an already established group structure. Additionally, we predict that the larger a group is, the lower its cohesion will be (fifth hypothesis), as intimacy and feelings of closeness are more difficult to achieve in larger groups, as past research has demonstrated (Carron \& Spink, 1995; Wheelan, 2009; Widmeyer, Brawley, \& Carron, 1990). The more people are added to a group, the harder it becomes for individual group members to perceive the group as one instead of several subgroups. We also predict that increased group size will lead to larger group openness (sixth hypothesis), as the increased diversity will once again make outside changes more likely to be perceived as an asset and less likely to be perceived as a threat, given that members with "unusual" characteristics are more likely to already be part of the group.

To reliably assess the new constructs, we needed the teams in which we administered our questionnaire to meet a few requirements. To ensure clear team affiliation, we looked for an organization with several small, clear-cut teams (and without members who switch frequently between teams). The members of these teams also needed to frequently interact with one another and work together interdependently so that each team member is able to reliably judge their team. One profession that meets these requirements is nursing in a hospital setting; thus, we conducted our study with the nursing staff of two university hospitals.

\section{Method}

\subsection{Sample}

Our sample consisted of 399 nurses at two university hospitals in Cologne and Bonn, Germany. $84.3 \%$ of the nurses were female, $14.2 \%$ were male, and $1.5 \%$ did not indicate their gender. The first hospital yielded 207 participants (referred to hereafter as the "Cologne sample"), while the second yielded 192 (referred to hereafter as the "Bonn sample"). Age within the samples ranged from 20 to 62 $(M=35.8, S D=11.31)$. On average, each team consisted of 21.4 members, of which a mean of $39.5 \%$ were assessed. All participants in the study participated voluntarily in a larger research project that the administration of the questionnaire presented here was a part of.

\subsection{Procedure}

The questionnaires were filled out either at the beginning of a professional development training or during normal working hours whenever the situation permitted doing so. To calculate the test-retest reliability of the instrument, 35 
nurses filled out the questionnaire twice, with the timeframe between administrations ranging from 2 to 11 weeks.

\subsection{Measures}

For group openness, we formulated items asking each team member for an assessment of their entire team, in accordance with the direct approach described in the introduction. Existing items for the openness subscale at the individual level, such as "Sometimes I just like to watch the wind as it blows through the trees" (Ashton \& Lee, 2007), certainly make sense to answer at the individual level, but summarizing these answers across individuals cannot logically be expected to yield group behavior or group personality. In fact, we suggest that behavioral patterns of openness at the group level are slightly different from those of openness at the individual level. Whereas the latter manifests itself in behaviors such as visiting an art gallery or travelling more often, at the group level, the resulting behaviors and characteristics are more basal, such as welcoming new members in a friendly and encouraging manner or asking fellow group members for advice. Therefore, we measured what we conceptualized as group openness with items in which individual members had to rate their entire group (in the case of this study, their work teams). Example items are: "New ideas are considered in our team" and "The team is open to changes".

For cohesion, new items were created with our more general explanation of the construct in mind to avoid formulating items that only fit a specific group, which would have gone against the notion of a general group personality instrument independent of any specific assessed group. As was the case for group openness, the cohesion items were formulated in a way that asked each individual to evaluate their entire team, in accordance with the direct approach. Example items are: "In our team, we have a 'we're all in the same boat' attitude" and "In our team, problems can be freely brought up".

The items we generated formed the initial version of the Group Openness and Cohesion Questionnaire, which consisted of 9 group openness items and 11 cohesion items (see Appendix A for the items and translations and Appendix B for the questionnaire as it was used in the research project). All items were answered on a 5-point Likert scale, with answer alternatives worded "totally agree", "mostly agree", "somewhat agree", "agree a little bit", and "do not agree at all". All items were in German.

Aside from administering the initial version of the Group Openness and Cohesion Questionnaire, we assessed participants' gender and age, as well as how many members their team consists of and the duration of their team membership so far.

\section{Results}

\subsection{Internal Structure}

Our first goal was to find a final version of the Group Openness and Cohesion 
Questionnaire. In order to do so, we tested the full instrument with the Cologne sample using confirmatory factor analysis (CFA) through a structural equation model, made adjustments to the instrument on the basis of the CFA results, and finally cross-validated the final instrument with the Bonn sample.

To assess model fit, we used a combination of common fit indices. For the Root Mean Square Error of Approximation (RMSEA) and Comparative Fit Index (CFI), we followed the cutoff criteria suggested by Hu \& Bentler (1999); for the Goodness of Fit Index (GFI) and the Adjusted Goodness of Fit Index (AGFI), we followed the recommendations of Jöreskog (Jöreskog, 1993). If we obtained an unsatisfactory model fit, we planned to use the modification indices and factor loadings to decide which items to delete and which covariances between item errors to allow. We predetermined factor loadings of .40 or less to be unsatisfactory; absolute cutoff values for modification indices are not possible to predetermine, as these indices can only be interpreted relative to each other.

Generally speaking, the Cologne sample was used to test and modify the model. To ensure that the improvements in fit shown by the modified model were not due to capitalization on chance, the model was cross-validated with the second data set (Bonn sample).

When testing model 1 for the full instrument (two latent variables, group openness and cohesion, with 9 resp. 11 measured variables loading onto those latent variables) on the Cologne sample, we noticed a very high correlation of .80 between the latent factors openness and cohesion. Therefore, we tested how a model with only one latent factor instead of the proposed two fit the data. In the model with only one latent factor, all items were assigned to that one factor, while in the two-factor model, each item was assigned to its respective factor (openness or cohesion). We used the comparative model fit indices Akaike Information Criterion (AIC) and Bayesian Information Criterion (BIC) to compare these two models, which revealed the clear superiority of the solution with two latent factors: The model with one latent factor had an AIC value of 649.61 and a BIC value of 782.92 associated with it, while the model with two latent factors had an AIC value of 472.38 and a BIC value of 608.88 associated with it. AIC and BIC values cannot be interpreted individually and only serve to compare two models to one another. As the model with smaller AIC and BIC values always fits the data better, the model with one latent factor was rejected. As these models are not nested, only AIC and BIC are reported (comparing $\chi^{2}$ statistics and fit indices that rely on those is not permissible). All further analyses were carried out with the two-factor model.

The RMSEA of the initial instrument with the Cologne sample was .08 and the Comparative Fit Index (CFI) was .907; thus, both showed room for improvement. For a full list of fit indices and associated results, see Table 1. To improve model fit, the modification indices called for the removal of Item 10 (originally part of the openness factor), which covaried to a larger degree with the cohesion factor than it did with the openness factor (M.I. for cohesion: 26.440, M.I. for 
Table 1. Fit indices from both the initial version of the instrument with 20 items (first row), and the adjusted instrument (second and third rows), i.e., the final version of the instrument with 17 items.

\begin{tabular}{lcccccccc}
\hline \multicolumn{1}{c}{ Model } & $\chi^{2}$ & DF & p & GFI & AGFI & RMSEA & PClose & CFI \\
\hline $\begin{array}{l}\text { Model 1: Initial instrument } \\
\text { (Cologne sample) }\end{array}$ & 390.238 & 169 & $<.001$ & .843 & .805 & .080 & .000 & .907 \\
$\begin{array}{l}\text { Model 2a: Final instrument } \\
\text { (Cologne sample) }\end{array}$ & 205.633 & 117 & $<.001$ & .897 & .865 & .061 & .100 & .956 \\
$\begin{array}{l}\text { Model 2b: Final instrument } \\
\text { (Bonn sample) }\end{array}$ & 223.805 & 117 & $<.001$ & .871 & .831 & .069 & .013 & .945 \\
\hline
\end{tabular}

openness 17.205). Translated, the item reads, "In our team, we support each other with the implementation of new ideas", which in retrospect obviously fits the underlying ideas behind both constructs rather than that of openness only. This led to the item's exclusion. Additionally, a modification index of 33.932 suggested allowing the error variances of Item 14 and Item 16 to covary. Doing so made sense with regard to the content of the items as well: Translated, Item 14 reads, "In our team, we have a 'We have always done it this way' attitude", and Item 16 reads, "Changes to our ways of working are blocked in our team". These items have a specific "active blocking of changes in work processes" factor in common that exceeds the generic group openness factor. All other modification indices were very small in relative value (all < 15.746) and therefore did not lead to further adjustments. Finally, Items 02 (translated: "My team has a difficult time deviating from routine.") and 13 (translated: "Personal things are sometimes discussed in the team.") were excluded because of factor loadings of .40 or less. In sum, the version of the questionnaire where Item 10 was deleted, the errors of Item 14 and Item 16 were covaried, and Items 02 and 13 were deleted was selected as the final version, as all further deletions and adjustments did not improve model fit. The model resulting from these modifications (with 7 measured variables loading on openness and 10 measured variables loading on cohesion) is referred to as Model 2a (see Table 1). Although $\chi^{2}$ is significant, the other fit indices indicate that the model now provides reasonable fit to the data.

The final version of the instrument was then cross-validated with the Bonn sample, again using a confirmatory factor analysis through a structural equation model. All fit indices are reported in Table 1 as Model $2 \mathrm{~b}$. The model of the final instrument along with the factor loadings, the between-factor correlation and the measurement errors obtained in the Bonn sample are shown in Figure 1. As indicated by the Fit indices in Table 1, the instrument is stable across samples.

\subsection{Psychometric Properties of the Final Instrument}

Item and scale statistics were also calculated for both samples (using the final instrument). These are shown in Table 2.

Taken together, the CFA results and psychometric properties of the final version of the instrument confirm that the response structure of our results fits the 


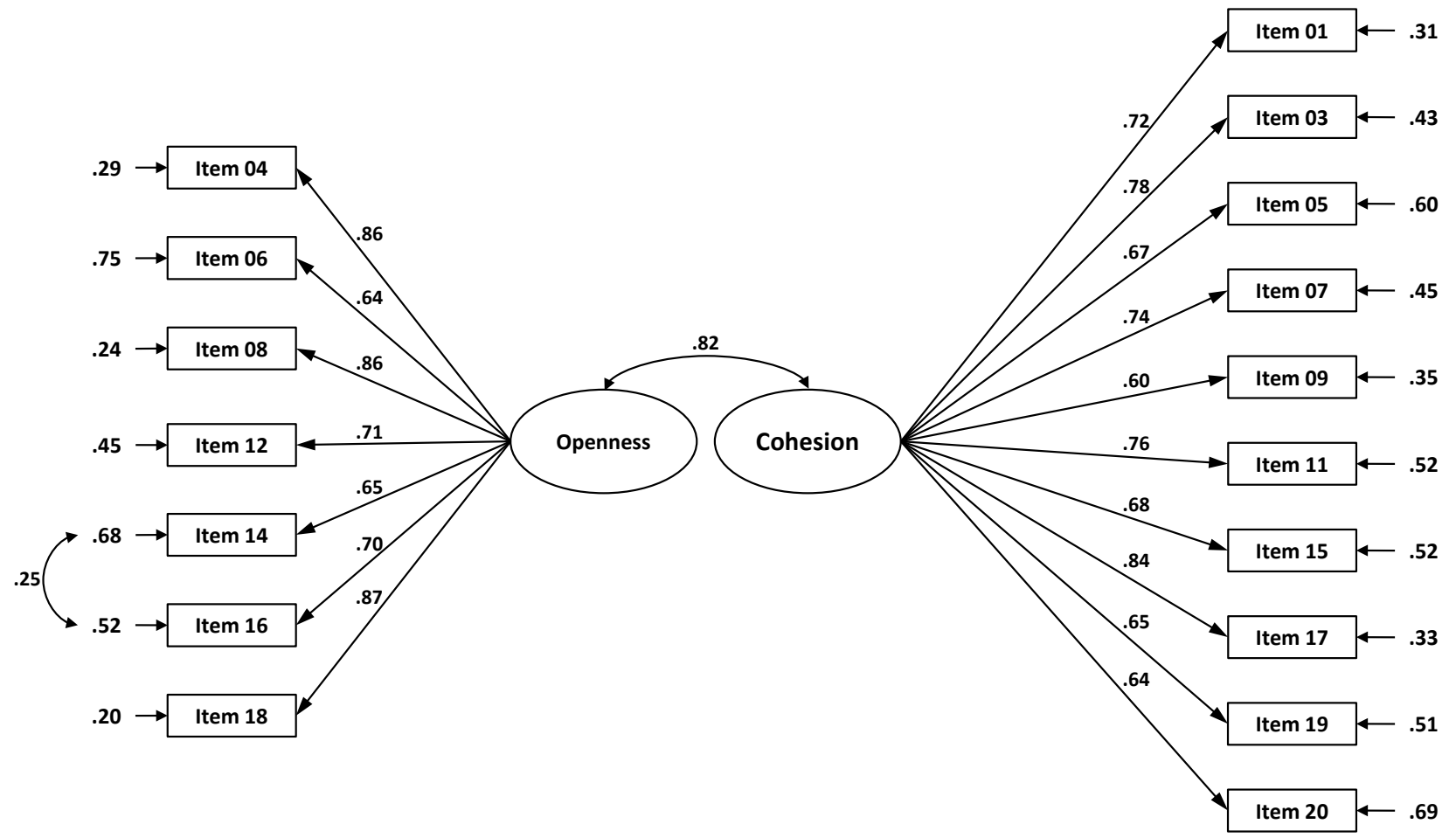

Figure 1. The final model after all adjustments is displayed here, along with the factor loadings, the between-factor correlation and measurement errors obtained in the Bonn sample. For Items 14 and 16, the covariance between their respective errors is displayed as well.

Table 2. Psychometric properties of the items in the final instrument separated by sample. Means and standard deviations for both scales and their respective items, item-scale correlations and item difficulties for the items only.

\begin{tabular}{|c|c|c|c|c|c|c|c|c|}
\hline \multirow{3}{*}{$\begin{array}{c}\text { Scale/Item } \\
\text { Cohesion }\end{array}$} & \multicolumn{4}{|c|}{$M(S D)$} & \multicolumn{2}{|c|}{$r_{i t}$} & \multicolumn{2}{|c|}{$p_{i}$} \\
\hline & \multicolumn{2}{|c|}{ Cologne } & \multicolumn{2}{|c|}{ Bonn } & \multirow[t]{2}{*}{ Cologne } & \multirow[t]{2}{*}{ Bonn } & \multirow[t]{2}{*}{ Cologne } & \multirow[t]{2}{*}{ Bonn } \\
\hline & 3.35 & $(.77)$ & 3.54 & $(.73)$ & & & & \\
\hline Item01 & 3.65 & (.85) & 3.70 & $(.80)$ & .71 & .67 & .66 & .68 \\
\hline Item03 & 3.26 & $(1.05)$ & 3.46 & $(1.06)$ & .78 & .73 & .56 & .62 \\
\hline Item05 & 2.83 & $(1.16)$ & 3.01 & $(1.04)$ & .69 & .63 & .46 & .50 \\
\hline Item07 & 2.94 & $(1.00)$ & 3.34 & $(1.00)$ & .76 & .70 & .48 & .58 \\
\hline Item09 & 3.99 & $(.77)$ & 4.24 & $(.74)$ & .59 & .57 & .75 & .81 \\
\hline Item 11 & 3.08 & $(1.02)$ & 3.38 & $(1.10)$ & .71 & .73 & .52 & .59 \\
\hline Item 15 & 3.30 & $(.98)$ & 3.46 & $(.98)$ & .76 & .64 & .57 & .62 \\
\hline Item17 & 3.64 & $(1.07)$ & 3.75 & (1.07) & .80 & .81 & .66 & .69 \\
\hline Item 19 & 3.50 & $(.91)$ & 3.64 & (.95) & .57 & .61 & .63 & .66 \\
\hline Item 20 & 3.31 & $(1.17)$ & 3.41 & $(1.08)$ & .68 & .61 & .58 & .60 \\
\hline Openness & 3.23 & $(.72)$ & 3.29 & $(.81)$ & & & & \\
\hline Item04 & 3.25 & $(.93)$ & 3.37 & $(1.05)$ & .73 & .80 & .56 & .59 \\
\hline Item06 & 3.06 & $(1.00)$ & 3.07 & $(1.13)$ & .71 & .63 & .51 & .52 \\
\hline Item 08 & 3.14 & $(.91)$ & 3.22 & $(.96)$ & .76 & .81 & .54 & .56 \\
\hline Item 12 & 3.04 & (.88) & 3.15 & $(.96)$ & .62 & .64 & .51 & .54 \\
\hline Item 14 & 3.32 & $(.98)$ & 3.25 & $(1.09)$ & .58 & .66 & .58 & .56 \\
\hline Item 16 & 3.59 & $(.87)$ & 3.60 & $(1.01)$ & .67 & .70 & .65 & .65 \\
\hline Item 18 & 3.22 & (.88) & 3.37 & $(.91)$ & .74 & .79 & .56 & .59 \\
\hline
\end{tabular}

Note: Cronbach's $\alpha$ for the full Cohesion scale was .921 in the Cologne sample and .908 in the Bonn sample. Cronbach's $\alpha$ for the full Group Openness scale was .891 in the Cologne sample and .903 in the Bonn sample. 
instrument's expected structure. From here on, the final version of the instrument will be referred to as the Group Openness and Cohesion Questionnaire (GOCQ).

\subsection{Test-Retest Reliability}

To assess the test-retest reliability of the GOCQ, the questionnaire was administered twice to a group of $N=35$ participants in the Cologne sample with the amount of time in between administrations ranging from 2 to 11 weeks. Test-retest reliability was very good for the cohesion scale $\left(r_{t t}=.84\right)$ and good for the openness scale $\left(r_{t t}=.72\right)$.

\subsection{Validity}

Validity was assessed using the full sample (Cologne and Bonn). Participants were asked how long they had been a member of their respective team as well as how many members their team consisted of during the investigation. This information was used to provide first hints of the validity of the group openness and cohesion-scales (see above). Team size and average membership time were categorized into three categories each.

When creating the three categories for team size, each team was assigned the median of what participants from this team had entered as their team size. The median was chosen instead of the arithmetic mean to cancel out the influence of outliers, who either overestimated or underestimated the size of their teams considerably. This resulted in 15 small teams ( 7 to 13.5 members), 18 medium-sized teams ( 15 to 22.5 members) and 15 large teams ( 24 to 60 members).

The average membership time on a team was used as a proxy for team age, which refers to how long team members had known each other and how long they had been working together at the point at which the questionnaire was administered. In order to ensure the reliability of this proxy measure, only teams in which more than $50 \%$ of team members filled out the instrument were included into calculations involving average membership time. In order to identify these teams, once again the median number of members given by the members of each team was used. This led to the identification of 18 teams in which more than $50 \%$ of members were surveyed. These teams were divided into three categories according to average membership time, resulting in 5 young teams ( 22.7 to 54.5 months), 8 medium-aged teams (60.5 to 97.6 months) and 5 old teams (112.7 to 170 months).

To validate the group openness and cohesion scales with team age, two ANOVAs were calculated with categorized mean team membership time (as a proxy for team age) as the independent variable and the scale mean of the cohesion and group openness scales, respectively, as the dependent variable. The first one-way ANOVA revealed a significant difference in cohesion depending on mean membership time, $F(2,192)=4.622, p<.05, \eta^{2}=.046$. Bonferroni-corrected post-hoc comparisons showed that while there was no significant 
difference between young and medium-age teams $(p=.996)$, the difference between old teams and medium-age teams $(p=.014)$ as well as the difference between young teams and old teams $(p=.022)$ turned out to be significant. The second one-way ANOVA showed a significant influence of team membership time on openness as well, $F(2,192)=3.7, p<.05, \eta^{2}=.037$. Bonferroni-corrected post-hoc comparisons showed that only the difference between old teams and medium-age teams was significant $(p=.02)$. Our expectation that older teams will have greater cohesion was partially supported, although the difference between young and medium-age teams turned out to be insignificant. The predicted influence of team age on group openness was also partially supported, with old teams being significantly more open than medium-age teams, but all other differences failed to reach significance.

To validate the group openness and cohesion scales with team size, two more ANOVAs were calculated with categorized median team size as the independent variable and the scale mean of the cohesion and team openness scales, respectively, as the dependent variable. The first one-way ANOVA revealed a significant influence of team size on cohesion, $F(2,395)=3.389, p<.05, \eta^{2}=.017$. Bonferroni-corrected post-hoc comparisons showed that only medium-sized teams and large teams differed significantly in cohesion $(p=.026)$. The second one-way ANOVA revealed no significant differences in openness depending on team size, $F(2,395)=1,894$, n.s. Therefore, our expectation that larger teams are less cohesive was partially supported, as large teams were significantly less cohesive than medium teams, but team size had no influence on team openness in this sample.

For an overview of the means and standard deviations of the cohesion and group openness scale means depending on team size and team age, see Table 3.

\subsection{Team Differentiation and Relevance of the Team Level}

We expected that individuals' ratings of team cohesion and team openness would be reflective of these characteristics at the team level. Therefore, we analyzed whether team membership served as a relevant predictor of individuals' experience of cohesion and openness within the team.

Teams were only included in the calculations if (A) at least 10 persons per team answered the questionnaire, and (B) team size was no greater than $N=20$

Table 3. Means (Standard deviations) of cohesion and group openness depending on team age and team size.

\begin{tabular}{ccccccccc}
\hline \multirow{2}{*}{ Scale } & \multicolumn{3}{c}{ Team Age } & & \multicolumn{3}{c}{ Team Size } \\
\cline { 2 - 3 } \cline { 7 - 8 } & Young & Medium & Old & & Small & Medium & Large \\
\hline \multirow{2}{*}{ Cohesion } & 3.342 & 3.353 & 3.774 & & 3.450 & 3.564 & 3.336 \\
& $(.102)$ & $(.079)$ & $(.125)$ & & $(.075)$ & $(.066)$ & $(.058)$ \\
Group- & 3.259 & 3.164 & 3.558 & & 3.303 & 3.288 & 3.192 \\
Openness & $(.101)$ & $(.077)$ & $(.123)$ & & $(.094)$ & $(.086)$ & $(.116)$ \\
\hline
\end{tabular}


(so that at least $50 \%$ of the team was represented by the $10+$ persons). The final sample consisted of 245 participants belonging to 18 teams. To check whether team membership was a relevant predictor of individual experience of cohesion and openness, intraclass coefficients (ICC) were calculated. ICC coefficients refer to differences between teams, i.e., represent the proportion of variance in cohesion and openness between teams. ICC were .27 for cohesion and .20 for openness, indicating that $27 \%$ and $20 \%$, respectively, of the variance in these two constructs is located at the team level. This means that the remaining $73 \%$ and $80 \%$ of variance in cohesion and openness, respectively, occurs at the individual level.

As a second way to analyze the relevance of the team level, Euclidean distances between each person's location (the combination of the person's individual scores for cohesion and openness) and the 18 team centers (the combination of each team's means in cohesion and openness) were calculated, resulting in 18 distance measures per person. We then determined two indices for each person: the distance between the person's location and their own team center (disteam), and the mean distance between the person's location and the other 17 team centers (disother). Using a within-subject ANOVA, significantly greater Euclidean distances to the other team centers (disother: $M=1.01, S D=0.48$ ) than to one's own team center (disteam: $M=.74, S D=.46$ ) were found, with $F(1,244)=$ $119.46, p<.001, \eta^{2}=.33$. In sum, both analyses show clear evidence that team membership is a relevant predictor of individuals' ratings of their team's cohesion and openness, in line with our expectations.

\section{Discussion}

The aims of this paper were to provide a theoretical framework for personality at the group level with two group personality dimensions, introduce a measurement instrument to assess these dimensions, and provide evidence for the validity of this instrument.

A clear concept of personality at the group level that could be applied to different groups independent of their context and setting has been noticeably absent from psychology. In this paper, we suggested group openness and cohesion as two basic dimensions of group personality.

Group openness, the first dimension of group personality, describes the outward orientation of a group. It refers to a group's ability and willingness to adapt to outside influences, such as new members, new ways of working and of working together, and new information and knowledge. Many studies have tried to assess groups' openness using the personalities of individual group members in order to predict outcomes such as group performance (Barrick et al., 1998; Homan et al., 2008). While this approach obviously lacks a justifiable rationale, the concept of openness at the group level continues to be a relevant characteristic of a group or team.

Cohesion, the second dimension of group personality, is a construct describing 
the inward orientation of a group, including the extent to which a group shares a common goal, how affectionate the group members are toward one another, how much they support each other, and to what extent the group experiences itself as a "unity". Cohesion is often an implicit sub-facet or subscale in inventories measuring some form of group climate, such as work team climate (Anderson \& West, 1998), family climate (Bjornberg \& Nicholson, 2007), or therapy group climate (Law et al., 2012). In fact, it can be argued that a collection of people needs to experience a certain degree of cohesion before it can be classified as a group.

In this study, we created a questionnaire to assess these two dimensions, cohesion and group openness, at the group level, with each member rating their group on each item instead of rating themselves only. The resulting Group Openness and Cohesion Questionnaire (GOCQ) was validated using CFA in two independent samples and yielded satisfactory to excellent model fit indices.

The correlation between the two latent factors cohesion and group openness turned out to be higher than expected. Of course, cohesion and group openness are similar, as both use the group as a reference point. Furthermore, ratings for both dimensions may be influenced by the rater's experience of the group's general functioning, which may account for the large intercorrelation. In the present samples taken from a hospital setting in which groups (teams) are non-competitive, we assume that combinations of high cohesion and high openness are beneficial for the success of teams. Other combinations, such as teams high in cohesion and low in openness, are more likely to be found in competitive environments such as finance or creative work, where disregarding other teams or outside ideas might be beneficial for workflow and success. Therefore, it seems necessary to examine in future research whether the high correlation found in our study also occurs in other types of groups, and to analyze which kinds of combinations are beneficial for which kinds of groups.

The validity of the GOCQ was analyzed using team size and team age. Regarding team size, large teams had significantly lower scores on cohesion than medium-sized teams, while all other differences were non-significant. In fact, of the three team sizes, large teams showed the smallest mean cohesion. Past research on group size and cohesion found similar results (Carron \& Spink, 1995; Wheelan, 2009; Widmeyer, Brawley, \& Carron, 1990). A possible explanation for this finding is that each team member took the behavior of all team members into consideration when rating the cohesion of his or her respective team. It follows that the larger the team, the more differentiated the behavior of the people within the team becomes, and the more diverse opinions and behaviors within the team become, the less likely team members will be to perceive their team as one cohesive entity. In other words, once a team reaches a certain size, it becomes much harder for an individual member to even perceive the team as a coherent group of people with similar behaviors and attitudes, which likely leads to the team to being perceived as less cohesive. 
For team age, which was categorized into young teams, medium-aged teams, and old teams, significant differences were found regarding both cohesion and openness. With respect to the former, old teams showed significantly higher cohesion than both medium-aged teams and young teams, while the latter two did not differ significantly. This indicates that cohesion increases with the longer a team's members have been part of the team (on average). This aligns with the idea of cohesion being, to a large extent, defined by interpersonal attraction and the presence of common goals within a group, as these parameters can reasonably be expected to increase as a team "ages together". A group's cohesion increases as differences are set aside and relationships are fostered, as is also reported by Harrison, Price, \& Bell (1998). Similarly, team openness increases with age as well, although the results are not as clear-cut here: old teams score significantly higher on openness than medium-aged teams, while all other differences are non-significant. It seems that there is a certain threshold in team age that, once reached, leads to a significant increase in openness. It might be that this process is similar to that with cohesion: the older a team is, the less changes (such as new members, new ways of working, etc.) are perceived as a disruption and the more they are welcomed, which is the quintessence of group openness. High team openness therefore seems to be found more in older teams. All of these findings about the relations between cohesion/team openness and team size or team age support the validity of the scales.

Since the GOCQ assesses constructs at the team level, we were greatly interested in confirming that team membership affects response tendencies for individuals, i.e., whether the instrument can differentiate between teams. This was done by calculating intraclass correlation coefficients, which revealed that $27 \%$ of the response variance in cohesion and $20 \%$ of the response variance in openness is located at the team level. This indicates that the individual perspective still plays a very large role as a reference point when answering the items, even when items are formulated in a way that asks respondents to evaluate their entire team. However, the other team-level analysis, comparing the Euclidian distances between each respondent and their own team vs. all other teams showed that participants were significantly closer to their own team than to all other teams in their response structure. We are therefore confident in saying that the instrument does indeed represent the response tendencies of entire teams.

The limitations of this study include the large variation in team size and proportion of team members examined. Additionally, this study did not assess personality at the individual level. A direct comparison between the dimensions proposed here and the well-established individual-level dimensions of the Five-Factor- or HEXACO-models (Ashton \& Lee, 2007; McCrae \& Costa Jr., 1999) would have shed additional light on the validity of cohesion and group openness as group personality dimensions. In future research, it would be fruitful to compare what we have labeled the direct and the indirect approaches to assessing group personality. For example, it would be interesting to see whether 
group openness really does have more predictive power than mean or maximum individual openness for outcomes such as team performance or acceptance of trainings.

\section{Conclusion}

Overall, this study took a further step towards conceptualizing and measuring personality at the group level. Through the creation and administration of a questionnaire measuring the two dimensions group openness and cohesion in a hospital setting with multiple work teams, we aimed to continue closing the gap in personality research on the personality of groups. This study was successful in showing that group openness and cohesion are meaningful constructs that can be measured at the group level using the presented questionnaire GOCQ, which was created for this study. This study also provides insight into how group size and group age affect members' perception of group openness and cohesion. The main contributions of this paper to the literature of group personality are the division and description of the indirect and the direct approach to measuring group personality constructs (and other group traits), the development of a theory regarding the structure of personality at the group level, and the successful application of this theory to practice by measuring two dimensions of group personality in group openness and cohesion. More studies comparing this approach to the individual-level personalities of group members and assessing correlates with and outcomes of group personality are called for.

\section{Fund}

This work was supported by the Federal Ministry of Education and Research (BMBF) [grant number: 02L14A150]. The BMBF was not involved in study design, data collection, data analysis, data interpretation, and in the writing of this report.

\section{References}

Anderson, N. R., \& West, M. A. (1996). The Team Climate Inventory: Development of the TCI and Its Applications in Teambuilding for Innovativeness. European Journal of Work and Organizational Psychology, 5, 53-66. https://doi.org/10.1080/13594329608414840

Anderson, N. R., \& West, M. A. (1998). Measuring Climate for Work Group Innovation: Development and Validation of the Team Climate Inventory. Journal of Organizational Behavior, 19, 235-258. https://doi.org/10.1002/(SICI)1099-1379(199805)19:3<235::AID-JOB837>3.0.CO;2-C

Ashton, M. C., \& Lee, K. (2007). Empirical, Theoretical, and Practical Advantages of the HEXACO Model of Personality Structure. Personality and Social Psychology Review, 11, 150-166. https://doi.org/10.1177/1088868306294907

Barrick, M. R., \& Mount, M. K. (1991). The Big Five Personality Dimensions and Job Performance: A Meta-Analysis. Personnel Psychology, 44, 1-26.

https://doi.org/10.1111/j.1744-6570.1991.tb00688.x 
Barrick, M. R., Stewart, G. L., Neubert, M. J., \& Mount, M. K. (1998). Relating Member Ability and Personality to Work-Team Processes and Team Effectiveness. Journal of Applied Psychology, 83, 377-391. https://doi.org/10.1037/0021-9010.83.3.377

Barry, B., \& Stewart, G. L. (1997). Composition, Process, and Performance in Self-Managed Groups: The Role of Personality. Journal of Applied Psychology, 82, 62-78. https://doi.org/10.1037/0021-9010.82.1.62

Bear, M., Oldham, G. R., Jacobsohn, G. C., \& Hollingshead, A. B. (2008). The Personality Composition of Teams and Creativity: The Moderating Role of Team Creative Confidence. The Journal of Creative Behavior, 42, 255-282. https://doi.org/10.1002/j.2162-6057.2008.tb01299.x

Bjornberg, A., \& Nicholson, N. (2007). The Family Climate Scales: Development of a New Measure for Use in Family Business Research. Family Business Review, 20, 229-246. https://doi.org/10.1111/j.1741-6248.2007.00098.x

Bond, M. H., \& Shiu, W. Y. F. (1997). The Relationship between a Group's Personality Resources and the Two Dimensions of Its Group Process. Small Group Research, 28, 194-217. https://doi.org/10.1177/1046496497282002

Carron, A. V., \& Brawley, L. R. (2000). Cohesion: Conceptual and Measurement Issues. Small Group Research, 31, 89-106. https://doi.org/10.1177/104649640003100105

Carron, A. V., \& Spink, K. S. (1995). The Group Size-Cohesion Relationship in Minimal Groups. Small Group Research, 26, 86-105. https://doi.org/10.1177/1046496495261005

Carron, A. V., Widmeyer, W. N., \& Brawley, L. R. (1985). The Development of an Instrument to Assess Cohesion in Sport Teams: The Group Environment Questionnaire. Journal of Sport Psychology, 7, 244-266. https://doi.org/10.1123/jsp.7.3.244

Cheng, C., Bartram, T., Karimi, L., \& Leggat, S. G. (2013). The Role of Team Climate in the Management of Emotional Labour: Implications for Nurse Retention. Journal of Advanced Nursing, 69, 2812-2825. https://doi.org/10.1111/jan.12202

Dang, C. C., \& Ilgen, D. R. (2006). Team Personality: A Dynamic, Contextualized View. Zeitschriftfür Personalpsychologie, 5, 177-185. https://doi.org/10.1026/1617-6391.5.4.177

Eys, M. A., Lougheed, T., Bray, S. R., \& Carron, A. V. (2009). Development of a Cohesion Questionnaire for Youth: The Youth Sport Environment Questionnaire. Journal of Sport and Exercise Psychology, 31, 390-408. https://doi.org/10.1123/jsep.31.3.390

Harrison, D. A., Price, K. H., \& Bell, M. P. (1998). Beyond Relational Demography: Time and the Effects of Surface- and Deep-Level Diversity on Work Group Cohesion. Academy of Management Journal, 41, 96-107. https://doi.org/10.2307/256901

Heslin, R. (1964). Predicting Group Task Effectiveness from Member Characteristics. Psychological Bulletin, 62, 248-256. https://doi.org/10.1037/h0048870

Homan, A. C., Hollenbeck, J. R., Humphrey, S. E., Van Knippenberg, D., Ilgen, D. R., \& Van Kleef, G. A. (2008). Facing Differences with an Open Mind: Openness to Experience, Salience of Intragroup Differences, and Performance of Diverse Work Groups. Academy of Management Journal, 51, 1204-1222. https://doi.org/10.5465/AMJ.2008.35732995

Hu, L. T., \& Bentler, P. M. (1999). Cutoff Criteria for Fit Indexes in Covariance Structure Analysis: Conventional Criteria versus New Alternatives. Structural Equation Modeling: A Multidisciplinary Journal, 6, 1-55. https://doi.org/10.1080/10705519909540118

Jackson, S. E., Brett, J. F., Sessa, V. I., Cooper, D. M., Julin, J. A., \& Peyronnin, K. (1991). Some Differences Make a Difference: Individual Dissimilarity and Group Heterogeneity as Correlates of Recruitment, Promotions, and Turnover. Journal of Applied Psychology, 76, 675-689. https://doi.org/10.1037/0021-9010.76.5.675 
Johnson, D. W., \& Johnson, R. T. (1983). Social Interdepedence and Perceived Academic and Personal Support in the Classroom. The Journal of Social Psychology, 120, 77-82. https://doi.org/10.1080/00224545.1983.9712012

Jöreskog, K. G. (1993). Testing Structural Equation Models. In K. A. Bollen, \& J. S. Lang (Eds.), Testing Structural Equation Models (pp. 294-317). Newbury Park, CA: Sage.

Kenrick, D. T., \& Funder, D. C. (1988). Profiting from Controversy. Lessons from the Person-Situation Debate. American Psychologist, 43, 23-34. https://doi.org/10.1037/0003-066X.43.1.23

Kurdek, L. A., \& Fine, M. A. (1993). The Relation between Family Structure and Young Adolescents' Appraisal of Family Climate and Parenting Behavior. Journal of Family Issues, 14, 279-290. https://doi.org/10.1177/019251393014002007

Kurdek, L. A., Fine, M. A., \& Sinclair, R. J. (1995). School Adjustment in Sixth Graders: Parenting Transitions, Family Climate, and Peer Norm Effects. Child Development, 66, 430-445. https://doi.org/10.2307/1131588

Law, T., Lee, K. Y., Ho, F. N., Vlantis, A. C., van Hasselt, A. C., \& Tong, M. C. (2012). The Effectiveness of Group Voice Therapy: A Group Climate Perspective. Journal of Voice, 26, e41-e48. https://doi.org/10.1016/j.jvoice.2010.12.003

MacKenzie, K. R., \& Tschuschke, V. (1993). Relatedness, Group Work and Outcome in Long-Tern Inpatient Psychotherapy Groups. Journal of Psychotherapy Practice and Research, 2, 147.

McCrae, R. R., \& Costa Jr., P. T. (1999). A Five-Factor Theory of Personality. In L. A. Pervin, \& O. P. John (Eds.), Handbook of Personality: Theory and Research (Vol. 2, pp. 139-153). New York: Guilford Press.

Patterson, M., West, M., Shackleton, V., Dawson, J., Lawthom, R., Matlis, S. et al. (2005). Validating the Organizational Climate Measure: Links to Managerial Practices, Productivity and Innovation. Journal of Organizational Behavior, 26, 379-408. https://doi.org/10.1002/job.312

Sbicigo, J. B., \& Dell'Aglio, D. D. (2012). Family Environment and Psychological Adaption in Adolescents. Psicologia: Reflexão e Crítica, 25, 615-622.

https://doi.org/10.1590/S0102-79722012000300022

Schneider, B. (1987). The People Make the Place. PersonnelPsychology, 40, 437-453. https://doi.org/10.1111/j.1744-6570.1987.tb00609.x

Schneider, B. (2000). The Psychological Life of Organizations. In N. Ashkanasy, C. P. M. Wilderom, \& M. F. Peterson (Eds.), Handbook of Organizational Culture \& Climate (pp. 17-21). Thousand Oaks, CA: Sage.

Schneider, B., \& Reichers, A. E. (1983). On the Etiology of Climates. Personnel Psychology, 36, 19-39. https://doi.org/10.1111/j.1744-6570.1983.tb00500.x

Steiner, I. D. (1972). Group Process and Productivity. New York, NY: Academic Press.

Tsui, A. S., Egan, T. D., \& Oreilly, C. A. (1992). Being Different: Relational Demography and Organizational Attachment. Administrative Science Quarterly, 37, 549-579. https://doi.org/10.2307/2393472

Van Vianen, A. E. M., \& De Dreu, C. K. W. (2001). Personality in Teams: Its Relationship to Social Cohesion, Task Cohesion, and Team Performance. European Journal of Work and Organizational Psychology, 10, 97-120. https://doi.org/10.1080/13594320143000573

Wheelan, S. A. (2009). Group Size, Group Development, and Group Productivity. Small Group Research, 40, 247-262. https://doi.org/10.1177/1046496408328703 
Widmeyer, W., Brawley, L. R., \& Carron, A. V. (1990). The Effects of Group Size in Sport. Journal of Sport and Exercise Psychology, 12, 177-190.

https://doi.org/10.1123/jsep.12.2.177

Williams, W. M., \& Sternberg, R. J. (1988). Group Intelligence: Why Some Groups Are Better than Others. Intelligence, 12, 351-377.

https://doi.org/10.1016/0160-2896(88)90002-5

Zhu, X., Wholey, D. R., Cain, C., \& Natafgi, N. (2016). Staff Turnover in Assertive Community Treatment (Act) Teams: The Role of Team Climate. Administration and Policy in Mental Health and Mental Health Services Research. 